\title{
EDITORIAL
}

\section{Fifty years of TCA}

\author{
Christopher J. Cramer
}

Published online: 13 January 2012

(C) Springer-Verlag 2012

Most of us probably do not remember the appearance of Volume 1, Number 1 of Theoretica Chimica Acta (TCA); it was in 1962. The present editorial board members were all very young at that time, if they had even yet been born. TCA was the first journal devoted exclusively to theoretical chemistry. Since then many other theoretical chemistry journals have debuted, including Int. J. Quantum Chem., J. Comput. Chem., Computational and Theoretical Chemistry (formerly Theochem), and J. Chem. Theory Comput. Meanwhile, in 1996, TCA changed its name from the Latin Theoretica Chimica Acta to the English Theoretical Chemistry Accounts.

The golden jubilee of the first theoretical chemistry journal provides an occasion both to look back and to look forward. When TCA was inaugurated, quantum mechanics was 37 years old. Now, it is 87 years old. When TCA was inaugurated, J. Chem. Phys. was 29 years old, Phys. Rev. as a journal of the American Physical Society was 49 years old, and J. Phys. Chem. was 66 years old. In the most recent impact factors (those for 2010), J. Chem. Phys., TCA, and Phys. Rev. A all have impact factors of 2.9, and J. Phys. Chem. A has an impact factor of 2.7. Of the 49 Nobel Prizes in chemistry since 1962 (the 2011 Prize has not been announced at the time we are putting this to press), theoretical chemists have shared or won six: Lars Onsager, William Lipscomb, Ilya Progogine, Kenichi Fukui and Roald Hoffman (shared), Rudy Marcus, and Walter Kohn

Published as part of the special collection of articles celebrating the 50th anniversary of Theoretical Chemistry Accounts/Theoretica Chimica Acta.

\section{J. Cramer ( $₫)$}

Department of Chemistry, University of Minnesota, 207 Pleasant Street SE, Minneapolis, MN 55455-0431, USA

e-mail: cramer@umn.edu and John Pople (shared). We feel safe predicting that theoretical chemists will take home more than six of the next 49 .

Let's look back in two ways. First, we wish to thank all those who have contributed to TCA through editorial service or editorial advisory service. Second, we wish to single out the most highly cited papers in the history of TCA. Then, we will look forward by means of the rest of this special issue.

\section{Editorial service}

Here is a list of those who have provided editorial service to TCA and the capacities in which they served. On behalf of the entire theoretical chemistry community, we thank them for their service.

\section{Honorary Editor}

Klaus Ruedenberg

\section{Editor-in-Chief}

Cramer, Christopher J.

Hartmann, Hermann

Ruedenberg, Klaus

Truhlar, Donald G.

\section{Editor or Associate Editor}

Baer, Roi

Cársky, Petr

Chandler, David

Cramer, Christopher J.

Davidson, Ernest R.

Handy, Nicholas C.

Iwata, Suehiro

Jortner, Joshua 


\section{Kutzelnigg, Werner}

Michl, Josef

Miller, William H.

Morokuma, Keiji

Roos, Björn O.

Salahub, Dennis R.

Schaefer III, Henry F.

Truhlar, Donald G.

$\mathrm{Wu}$, Yundong

\section{Chief Advisory Editor}

Truhlar, Donald G.

\section{Editorial Board}

Ahlrichs, Reinhart

Almlöf, Jan E.

Ángyán, János G.

Apeloig, Yitzhak

Aquilanti, Vincenzo

Åqvist, Johan

Baerends, Evert J.

Bagchi, Biman A.

Baldridge, Kim K.

Ballhausen, Carl J.

Barone, Vincenzo

Bartlett, Rodney J.

Basilevsky, Mikhail V.

Bauschlicher, Charles W.

Becke, Axel D.

Berne, Bruce J.

Bertrán, Juan

Botschwina, Peter

Brooks, Charles

Brown, Ronald D.

Bunge, Carlos F.

Cársky, Petr

Case, David A.

Catlow, Richard

Champagne, Benoit

Chelikowsky, James R.

Child, Mark S.

Clary, David C.

Collins, Michael A.

Davidson, Ernest R.

DiLabio, Gino A.

Dykstra, Clifford E.

Eisenstein, Odile

Elber, Ron

Field, Martin J.

Freed, Karl F.

Frenkel, Daan

Frenking, Gernot

Fried, Joel R.
Fukui, Kenichi

Gagliardi, Laura

Gale, Julian D.

Gao, Jiali

Garrett, Bruce C.

Gleiter, Rolf

Gordon, Mark S.

Grein, Fritz

Grimme, Stefan

Guo, Hua

Halevi, E. Amitai

Hall, George G.

Hall, Michael B.

Hammes-Schifffer, Sharon

Harvey, Jeremy

Hess, Bernd A.

Helgaker, Trygve

Heller, Eric J.

Hirao, Kimihiko

Hirata, So

Houk, Kendall N.

Iwata, Suehiro

Jordan, Kenneth D.

Jorgensen, William L.

Jortner, Joshua

Kaldor, Uzi

Kapral, Raymond

Karplus, Martin

Kato, Shigeki

Kaupp, Martin

Ketelaar, J. A. A.

Kollman, Peter

Kolos, Włodzimierz

Kotani, Masao

Kouri, Donald J.

Koutecky, Jaroslav

Kutzelnigg, Werner

Lavery, Richard

Levine, Raphael D.

Levy, Melvin P.

Lim, Carmay

Linnett, J. W.

Lluch, José M.

Luque, F. Javier

Madden, Paul A.

Malrieu, Jean-Paul

Marcus, Rudolph A.

Martin, Jan M. L.

Martinez, Todd J.

Mennucci, Benedetta

Merz, Kenneth M. Jr.

Meyer, Hans-Dieter

Meyer, Wilfried 
Miller, James A.

Miller, William H.

Morokuma, Keiji

Mukherjee, Debashis

Murcko, Mark

Nakatsuji, Hiroshi

Neckel, Adolf

Newton, Marshall D.

Nikitin, Evgueni E.

Noid, Donald W.

Oddershede, Jens

Olivucci, Massimo

Olsen, Jeppe

Ornellas, Fernando R.

Orozco, Modesto

Pacchioni, Gianfranco

Paldus, Josef

Parr, Robert G.

Parrinello, Michele

Pearson, Ralph G.

Petersson, George A.

Pettitt, B. Montgomery

Primas, Hans

Pullman, Bernard

Pyykko, Veli Pekka

Radom, Leo

Raghavachari, Krishnan

Ranby, B.

Ratner, Mark A.

Reiher, Markus

Robb, Michael A.

Roos, Björn O.

Rossky, Peter J.

Ruch, Ernst

Ruedenberg, Klaus

Salahub, Dennis R.

Sandorfy, Camille

Savin, Andreas

Schaefer III, Henry Fritz

Schatz, George C.

Schinke, Reinhard

Schlegel, H. Bernhard

Scuseria, Gustavo E.

Seideman, Tamar

Shaik, Sason

Shuai, Zhigang

Siegbahn, Per

Siepmann, J. Ilja

Simonetta, Massimo

Sinanoglu, Oktay

Skodje, Rex

Sprik, Michiel

Thiel, Walter
Thirumalai, Deverajan

Tomasi, Jacopo

Tucker, Susan C.

Tuckerman, Mark E.

Tully, John C.

van Santen, Rutger A.

van Wüllen, Christoph

Veillard, Alain

Voter, Arthur F.

Voth, Gregory A.

Warshel, Arieh

Werner, Hans-Joachim

Whangbo, Myung-Hwan

Yang, Jinlong

Yang, Weitao

Yarkony, David R.

Zahradnik, Rudolf

Zeng, Xiao Cheng

Zerner, Michael C.

Zhan, Chang-Guo

Zhang, John Z. H.

\section{Highly cited papers}

For each decade, the list contains the twelve most highly cited papers from that decade, as of the preparation of this editorial, with the most highly cited first. The lists contain many important contributors and many important contributions, and it is interesting to notice the evolution of topics represented therein. A key topic that has persisted on the lists for the full 50 years is basis sets, which have always been featured prominently in TCA.

2.1 First decade: 1962-1971 (Theoretica Chimica Acta)

- Some remarks on the Pariser-Parr-Pople method. Ohno, K. 1964. Theoretica Chimica Acta 2, pp. 219-227

- Possible "ferromagnetic states" of some hypothetical hydrocarbons. Mataga, N. Theoretica Chimica Acta 10 (4), pp. 372-376

- Recherches sur la géométrie de quelques hydrocarbures non-alternants: son influence sur les énergies de transition, une nouvelle définition de l'aromaticité. Julg, A., François, P. 1967 Theoretica Chimica Acta 8 (3), pp. 249-259

- Porphyrins XIV. Theory for the luminescent state in VO, Co, $\mathrm{Cu}$ complexes. Ake, R.L., Gouterman, M. 1969 Theoretica Chimica Acta 15 (1), pp. 20-42 60

- Gaussian basis set for molecular wavefunctions containing second-row atoms. Veillard, A. 1968 Theoretica Chimica Acta 12 (5), pp. 405-411 
- SCFMO calculations of heteroatomic systems with the variable $\beta$ approximation-I. Heteroatomic molecules containing nitrogen or oxygen atoms. Nishimoto, K., Forster, L.S. 1966 Theoretica Chimica Acta 4 (2), pp. $155-165$

- Porphyrins-VIII. Extended Hückel calculations on iron complexes. Zerner, M., Gouterman, M., Kobayashi, H. 1966 Theoretica Chimica Acta 6 (5), pp. 363-400

- Porphyrins-IV. Extended Hückel calculations on transition metal complexes. Zerner, M., Gouterman, M. 1966 Theoretica Chimica Acta 4 (1), pp. 44-63

- Valence orbital ionization potentials from atomic spectral data. Basch, H., Viste, A., Gray, H.B. 1965 Theoretica Chimica Acta 3 (5), pp. 458-464

- Energy partitioning with the CNDO method. Fischer, H., Kollmar, H. 1970 Theoretica Chimica Acta 16 (3), pp. 163-174

- The continuation of the periodic table up to $Z=172$. The chemistry of superheavy elements. Fricke, B., Greiner, W., Waber, J.T. 1971 Theoretica Chimica Acta 21 (3), pp. 235-260

- Electronic wave functions for atoms-II. Some aspects of the convergence of the configuration interaction expansion for the ground states of the He isoelectronic series. Bunge, C.F. 1970 Theoretica Chimica Acta 16 (2), pp. 126-144.

2.2 Second decade: 1972-1981 (Theoretica Chimica Acta)

- The influence of polarization functions on molecular orbital hydrogenation energies. Hariharan, P.C., Pople, J.A. 1973 Theoretica Chimica Acta 28 (3), pp. 213-222

- An intermediate neglect of differential overlap technique for spectroscopy: Pyrrole and the azines. Ridley, J., Zerner, M. 1973 Theoretica Chimica Acta 32 (2), pp. 111-134 1171

- Bonded-atom fragments for describing molecular charge densities. Hirshfeld, F.L. 1977 Theoretica Chimica Acta 44 (2), pp. 129-138

- On the calculation of bonding energies by the HartreeFock Slater method-I. The transition state method. Ziegler, T., Rauk, A. 1977 Theoretica Chimica Acta 46 (1), pp. 1-10

- Individualized configuration selection in CI calculations with subsequent energy extrapolation. Buenker, R.J., Peyerimhoff, S.D. 1974 Theoretica Chimica Acta 35 (1), pp. 33-58

- An intermediate neglect of differential overlap theory for transition metal complexes: $\mathrm{Fe}, \mathrm{Co}$ and $\mathrm{Cu}$ chlorides. Bacon, A.D., Zerner, M.C. 1979 Theoretica Chimica Acta 53 (1), pp. 21-54
- Triplet states via intermediate neglect of differential overlap: Benzene, pyridine and the diazines. Ridley, J.E., Zerner, M.C. 1976 Theoretica Chimica Acta 42 (3), pp. 223-236

- On the calculation of multiplet energies by the HartreeFock-Slater method. Ziegler, T., Rauk, A., Baerends, E.J. 1977 Theoretica Chimica Acta 43 (3), pp. 261-271

- Energy extrapolation in CI calculations. Buenker, R.J., Peyerimhoff, S.D. 1975 Theoretica Chimica Acta 39 (3), pp. 217-228

- A theoretical method to determine atomic pseudopotentials for electronic structure calculations of molecules and solids. Durand, P., Barthelat, J.-C. 1975 Theoretica Chimica Acta 38 (4), pp. 283-302

- Approximate calculation of the correlation energy for the closed shells. Colle, R., Salvetti, O. 1975 Theoretica Chimica Acta 37 (4), pp. 329-334

- Multiplicity of the ground state of large alternant organic molecules with conjugated bonds-(Do Organic Ferromagnetics Exist?). Ovchinnikov, A.A. 1978 Theoretica Chimica Acta 47 (4), pp. 297-304.

2.3 Third decade: 1982-1991 (Theoretica Chimica Acta)

- Energy-adjusted ab initio pseudopotentials for the second and third row transition elements. Andrae, D., Häußermann, U., Dolg, M., Stoll, H., Preuß, H. 1990 Theoretica Chimica Acta 77 (2), pp. 123-141

- Density matrix averaged atomic natural orbital (ANO) basis sets for correlated molecular wave functions-I. First row atoms. Widmark, P.-O., Malmqvist, P.Roos, B.O. 1990 Theoretica Chimica Acta 77 (5), pp. 291-306

- Medium-size polarized basis sets for high-level-correlated calculations of molecular electric properties-II. Second-row atoms: Si through Cl, Sadlej, A.J. 1991 Theoretica Chimica Acta 79 (2), pp. 123-140

- Energy-adjusted pseudopotentials for the rare earth elements. Dolg, M., Stoll, H., Savin, A., Preuss, H. 1989 Theoretica Chimica Acta 75 (3), pp. 173-194

- Density matrix averaged atomic natural orbital (ANO) basis sets for correlated molecular wave functions-II. Second row atoms. Widmark, P.-O., Persson, B.J., Roos, B.O. 1991 Theoretica Chimica Acta 79 (6), pp. 419-432

- $r_{12}$-Dependent terms in the wave function as closed sums of partial wave amplitudes for large 1. Kutzelnigg, W. 1985 Theoretica Chimica Acta 68 (6), pp. 445-469

- Orbital-invariant formulation and second-order gradient evaluation in Møller-Plesset perturbation theory. Pulay, P., Saebø, S. 1986 Theoretica Chimica Acta 69 (5-6), pp. $357-368$ 
- A generalized restricted open-shell Fock operator. Edwards, W.D., Zerner, M.C. 1987 Theoretica Chimica Acta 72 (5-6), pp. 347-361

- Theoretical investigations of molecules composed only of fluorine, oxygen and nitrogen: determination of the equilibrium structures of FOOF, (NO)2 and FNNF and the transition state structure for FNNF cis-trans isomerization. Lee, T.J., Rice, J.E., Scuseria, G.E., Schaefer III, H.F. 1989 Theoretica Chimica Acta 75 (2), pp. 81-98

- An overview of coupled cluster theory and its applications in physics. Bishop, R.F. 1991 Theoretica Chimica Acta 80 (2-3), pp. 95-148

- Recursive intermediate factorization and complete computational linearization of the coupled-cluster single, double, triple, and quadruple excitation equations. Kucharski, S.A., Bartlett, R.J. 1991 Theoretica Chimica Acta 80 (4-5), pp. 387-405

- A comparison of variational and non-variational internally contracted multiconfiguration-reference configuration interaction calculations. Werner, H.-J., Knowles, P.J. 1990 Theoretica Chimica Acta 78 (3), pp. 175-187.

2.4 Fourth decade: 1992-2001 (Theoretica Chimica Acta and Theoretical Chemistry Accounts)

- Towards an order- $N$ DFT method. Fonseca Guerra, C., Snijders, J.G., Te Velde, G., Baerends, E.J. 1998 Theoretical Chemistry Accounts 99 (6), pp. 391-403 1524

- Auxiliary basis sets for main row atoms and transition metals and their use to approximate Coulomb potentials. Eichkorn, K., Weigend, F., Treutler, O., Ahlrichs, R. 1997 Theoretical Chemistry Accounts 97 (1-4), pp. 119-124

- RI-MP2: First derivatives and global consistency. Weigend, F., Häser, M. 1997 Theoretical Chemistry Accounts 97 (1-4), pp. 331-340

- An efficient data compression method for the Davidson subspace diagonalization scheme Dachsel, H., Lischka, H. 1995 Theoretica Chimica Acta 92 (6), pp. 339-349

- An implementation of the conductor-like screening model of solvation within the Amsterdam density functional package. Pye, C.C., Ziegler, T. 1999 Theoretical Chemistry Accounts 101 (6), pp. 396-408

- Reparameterization of hybrid functionals based on energy differences of states of different multiplicity. Reiher, M., Salomon, O., Hess, B.A. 2001 Theoretical Chemistry Accounts 107 (1), pp. 48-55

- A combination of quasirelativistic pseudopotential and ligand field calculations for lanthanoid compounds. Dolg, M., Stoll, H., Preuss, H. 1993 Theoretica Chimica Acta 85 (6), pp. 441-450
- Density matrix averaged atomic natural orbital (ANO) basis sets for correlated molecular wave functions-III. First row transition metal atoms. Pou-Amérigo, R., Merchán, M., Nebot-Gil, I., Widmark, P.-O., Roos, B.O. 1995 Theoretica Chimica Acta 92 (3), pp. 149-181

- The singlet and triplet states of phenyl cation. A hybrid approach for locating minimum energy crossing points between non-interacting potential energy surfaces. Harvey, J.N., Aschi, M., Schwarz, H., Koch, W. 1998 Theoretical Chemistry Accounts 99 (2), pp. 95-99

- Internally contracted multiconfiguration-reference configuration interaction calculations for excited states. Knowles, P.J., Werner, H.-J. 1992 Theoretica Chimica Acta 84 (1-2), pp. 95-103

- Extensions and tests of "multimode": A code to obtain accurate vibration/rotation energies of many-mode molecules. Carter, S., Bowman, J.M., Handy, N.C. 1998 Theoretical Chemistry Accounts 100 (1-4), pp. 191-198

- The MIDI! basis set for quantum mechanical calculations of molecular geometries and partial charges. Easton, R.E., Giesen, D.J., Welch, A., Cramer, C.J., Truhlar, D.G. 1996 Theoretical Chemistry Accounts 93 (5), pp. 281-301.

2.5 Fifth decade: 2002-2011 (Theoretical Chemistry Accounts)

- The M06 suite of density functionals for main group thermochemistry, thermochemical kinetics, noncovalent interactions, excited states, and transition elements: Two new functionals and systematic testing of four M06-class functionals and 12 other functionals. Zhao, Y., Truhlar, D.G. 2008 Theoretical Chemistry Accounts 120 (1-3), pp. 215-241

- Systematically convergent basis sets for transition metals. II. Pseudopotential-based correlation consistent basis sets for the group $11(\mathrm{Cu}, \mathrm{Ag}, \mathrm{Au})$ and $12(\mathrm{Zn}, \mathrm{Cd}$, $\mathrm{Hg}$ ) elements. Peterson, K.A., Puzzarini, C. 2005 Theoretical Chemistry Accounts 114 (4-5), pp. 283-296

- Quantum molecular dynamics: Propagating wavepackets and density operators using the multiconfiguration time-dependent Hartree method. Meyer, H.-D., Worth, G.A. 2003 Theoretical Chemistry Accounts 109 (5), pp. 251-267 188

- QM/MM: What have we learned, where are we, and where do we go from here? Lin, H., Truhlar, D.G. 2007 Theoretical Chemistry Accounts 117 (2), pp. 185-199

- Ab initio calculation of molecular chiroptical properties. Crawford, T.D. 2006 Theoretical Chemistry Accounts 115 (4), pp. 227-245 
- Molecular potential-energy surfaces for chemical reaction dynamics. Collins, M.A. 2002 Theoretical Chemistry Accounts 108 (6), pp. 313-324

- Method of moments of coupled-cluster equations: A new formalism for designing accurate electronic structure methods for ground and excited states. Piecuch, P., Kowalski, K., Pimienta, I.S.O., Fan, P.-D., Lodriguito, M., McGuire, M.J., Kucharski, S.A., Kus, T., Musiał, M. 2004 Theoretical Chemistry Accounts 112 (5-6), pp. 349-393

- Investigation of the $\mathrm{S} 0 \rightarrow \mathrm{S} 1$ excitation in bacteriorhodopsin with the ONIOM(MO:MM) hybrid method. Vreven, T., Morokuma, K. 2003 Theoretical Chemistry Accounts 109 (3), pp. 125-132

- Electron localization function for transition-metal compounds. Kohout, M., Wagner, F.R., Grin, Y. 2002 Theoretical Chemistry Accounts 108 (3), pp. 150-156

- Improper, blue-shifting hydrogen bond. Hobza, P., Havlas, Z. 2002 Theoretical Chemistry Accounts 108 (6), pp. 325-334

- The fundamental nature and role of the electrostatic potential in atoms and molecules. Politzer, P., Murray, J.S. 2002 Theoretical Chemistry Accounts 108 (3), pp. 134-142

- Similarities and differences in the structure of 3d-metal monocarbides and monoxides. Gutsev, G.L., Andrews,
L., Bauschlicher Jr., C.W. 2003 Theoretical Chemistry Accounts 109 (6), pp. 298-308.

\section{A glimpse of the future: the 50th anniversary issue}

Each of our five guest editors has selected an important or emerging area of theoretical chemistry and has recruited experts to write about some key issues in that area. The five areas featured and their coordinators are:

- Electronic structure: present and future challenges (So Hirata)

- Relativistic quantum chemistry (Christoph van Wüllen)

- Reaction dynamics in gas phase and in solution (Hua Guo)

- Macromolecules and extended systems (Gino DiLabio)

- Spectroscopy, atmospheric chemistry, and thermochemistry (Fernando Ornellas)

We believe that the subjects of these featured articles encompass several areas that will be growing in importance over the next many years. We hope that you enjoy reading these contributions. 